\title{
Evaluación de la adecuación de las estancias en un hospital de tercer nivel
} Evaluation of the appropriateness of stays in a third level hospital

\author{
A. Pérez-Rubio, S. Santos, F. J. Luquero, S. Tamames, B. Cantón, J.J. Castrodeza
}

\section{RESUMEN}

\section{FUNDAMENTO}

The Appropriateness Evaluation Protocol (AEP) se ha mostrado como una herramienta útil para la revisión de la utilización de los recursos hospitalarios. El objetivo de este trabajo es conocer la proporción de ingresos y estancias inadecuadas, así como sus causas, en pacientes hospitalizados en el Hospital Clínico Universitario de Valladolid (HCUV).

\section{MATERIAL Y MÉTODOS}

Estudio observacional analítico de cohortes retrospectivo. El período de estudio ha sido de un año (2004). Se seleccionó una muestra de 1.630 ingresos. La definición de caso, las variables de interés y el modelo de recogida de datos se han llevado a cabo conforme al AEP. Se han analizado las principales variables mediante un análisis basal y las posibles relaciones entre ellas.

\section{RESULTADOS}

El 54\% de los ingresos presentaron al menos un día de estancia inadecuada, siendo la tasa de inadecuación global del $34,17 \%$. Entre las causas responsables de la inadecuación, el $68,9 \%$ de los ingresos presentaron al menos un criterio englobado dentro de la responsabilidad del médico o del hospital, y el $51,3 \%$ debido a retrasos en el desarrollo del estudio o tratamiento.

\section{CONCLUSIONES}

La utilización de métodos de identificación del uso inapropiado como el AEP presenta aplicaciones tanto en planificación como en gestión hospitalaria, al permitir identificar problemas hospitalarios causantes de demoras, principalmente problemas de tipo organizativo, permitiendo el desarrollo de intervenciones encaminadas a la reducción del uso inapropiado.

Palabras clave. Revisión de utilización. Utilización inadecuada de la hospitalización.

\begin{abstract}
BACKGROUND

The Appropriateness Evaluation Protocol (AEP) has proved to be a useful tool for reviewing the utilisation of hospital resources. The aim of this article is to determine the proportion of inappropriate admissions and stays, as well as their causes, in patients hospitalised in the Hospital Clínico Universitario de Valladolid (HCUV).
\end{abstract}

\section{MATERIAL AND METHODS}

A retrospective, analytical, observational, cohort study. The period of study was one year (2004). A sample of 1,630 admissions was gathered. Case definition, variables of interest and the model of data gathering were carried out in accordance with the AEP. The principal variables were analysed by means of a basal analysis and the possible relations between them.

\section{RESULTS}

Fifty-four percent of the admissions showed at least one day of inappropriate stay, with the global rate of inappropriateness being $34.17 \%$. Amongst the causes responsible for inappropriateness, $68.9 \%$ of admissions showed at least one criterion falling under the responsibility of the doctor or the hospital, and $51.3 \%$ were due to delays in the development of study or treatment.

\section{CONCLUSIONS}

The utilisation of methods of identification of inappropriate use such as AEP show applications both in planning and in hospital management, by making it possible to identify hospital problems causing delays, principally problems of an organisational type, making it possible to develop interventions aimed at reducing inappropriate use.

Key words. Review of utilisation. Inappropriate utilisation of hospitalisation.
Servicio de Medicina Preventiva y Salud Pública. Hospital Clínico Universitario de Valladolid

Aceptado para su publicación el 28 de septiembre de 2006

\section{Correspondencia:}

Alberto Pérez Rubio

Servicio de Medicina Preventiva y Salud Pública Hospital Clínico Universitario de Valladolid

C/ Ramón y Cajal, 3

47003 Valladolid

Tfno. 983420000 Ext 20129

Tfno móvil: 605156234

E-mail: albertoprz@gmail.com 


\section{INTRODUCCIÓN}

El aumento de la demanda sanitaria y la limitación de los recursos son la causa de un replanteamiento de la asistencia sanitaria, más acorde con la realidad socioeconómica del nuevo siglo XXI' ${ }^{1}$.

Aunque la estancia media ha mostrado una buena correlación con el consumo de recursos, no es un indicador válido de la estancia clínicamente necesaria para cada Grupo Relacionado de Diagnóstico ${ }^{2,3}$.

En 1981 se presentó un protocolo de revisión de utilización de los recursos sanitarios hospitalarios, The Appropriateness Evaluation Protocol (AEP) ${ }^{4,5}$, orientado a la identificación de días innecesarios de estancia hospitalaria sobre la base de la inexistencia de diversos procedimientos médicos, cuidados de enfermería o condiciones clínicas del paciente que justifiquen su permanencia en el hospital en un día concreto de la estancia hospitalaria. Este protocolo define como utilización inapropiada el uso de servicios hospitalarios cuando, desde el punto de vista estrictamente clínico, el paciente podría haber sido atendido en niveles asistenciales menos costosos o en menor tiempo. El $\mathrm{AEP}$, protocolo validado en España ${ }^{6}$, se presenta como el instrumento más conocido y utilizado, aunque no es el único. Se han desarrollado distintos instrumentos independientes para el diagnóstico de la utilización inapropiada como el ISD, SMI, DTO ${ }^{7}$.

Durante su desarrollo, el protocolo ha sufrido diversos cambios y mejoras, encaminados a clarificar su contenido y simplificar su aplicación para juzgar la adecuación de la presencia del paciente en el hospital $^{8}$.

La evaluación de la proporción de ingresos y estancias hospitalarias se ha realizado en múltiples hospitales, a través del empleo de los AEP, cuya validez y fiabilidad ha sido demostrada por evaluadores independientes en diversos países ${ }^{9,10}$. El AEP se ha mostrado como una herramienta útil para el conocimiento de las estancias e ingresos inadecuados de los pacientes en hospitales de agudos y de las causas que los determinan ${ }^{11,12}$.
El objetivo de este trabajo es conocer la proporción de ingresos y estancias inadecuadas, así como sus causas, en pacientes hospitalizados en el Hospital Clínico Universitario de Valladolid (HCUV).

\section{MATERIAL Y MÉTODOS}

Estudio observacional analítico de cohortes retrospectivo que se ha desarrollado en el Hospital Clínico Universitario de Valladolid (HCUV), un hospital de tercer nivel de titularidad pública perteneciente a la red asistencial de Salud de Castilla y León (SACYL). El período de estudio ha sido de un año y comprende del 1 de enero de 2004 al 31 de diciembre de 2004.

La población de estudio han sido los pacientes que ingresaron en el HCUV durante el citado período. De esta población se ha obtenido una muestra representativa, seleccionada de manera proporcional al número de ingresos que se producen en cada servicio. La muestra final estaba formada por 1.630 ingresos que originaron 16.183 estancias.

La definición de caso, las variables de interés y el modelo de recogida de datos se han llevado a cabo conforme al AEP.

La variable respuesta principal es la inadecuación tanto del ingreso como de la estancia hospitalaria. También se han evaluado las principales causas de la inadecuación. Se han considerado como variables explicativas la edad de los pacientes, el sexo, el tipo de ingreso (urgente o programado, el tipo de servicio (médico o quirúrgico) y el día que se produce la admisión.

Los datos se han obtenido mediante revisión de las historias clínicas de los pacientes que conforman la muestra.

Se ha realizado un análisis basal en el que se describen las principales variables. Un análisis crudo en el que se estudia la relación entre las distintas variables, utilizando la $\chi^{2}$ de Pearson para la comparación de variables discretas y la t de Student para la comparación de variables continuas. El análisis de los datos se ha realizado con el programa SPSS v13.0® y EPIDAT 3.0 para el cálculo de los riesgos 
relativos. Los intervalos de confianza se han estimado al $95 \%$.

\section{RESULTADOS}

En este estudio se han evaluado 1.630 ingresos correspondientes a 26 servicios, que han generado un total de 16.183 estancias.

\section{Descriptivo de la muestra}

La edad media de los pacientes fue de 62,5 años (DE= 18,03), siendo el $52,4 \%$ mayores de 65 años. Atendiendo a la distribución por sexo, el $58,5 \%$ fueron varones y el $41,5 \%$ mujeres.

Al diferenciar entre servicios médicos y quirúrgicos, se obtiene que el $43,6 \%$ corresponde a los primeros frente al 54,6\% que pertenecen a servicios quirúrgicos $\mathrm{y}$ en función de la procedencia del ingreso, el $50,2 \%$ fueron urgentes y el $49,8 \%$ programados. Ambas variables presentan diferencias estadísticamente significativas en su distribución por rangos de edad, predominando en personas mayores los ingresos en servicios médicos. Dentro de los servicios quirúrgicos el mayor porcentaje son ingresos programados, y en los médicos predominan los ingresos urgentes.

Considerando los días de estancia como una variable categórica, menor de 7 días y mayor de siete días, las primeras suponen un $53,9 \%$ de los ingresos frente al $47,1 \%$ de las mayores de una semana.

\section{Descriptivo de las estancias}

La estancia media ha sido de 9,9 días (DE= 9,66), y la mediana ha sido de 7 días.

El $62 \%$ del total de las estancias han sido ocasionadas por pacientes mayores de 65 años. Se observa de forma estadísticamente significativa que una mayor edad de los pacientes se relaciona con una mayor estancia hospitalaria. Los varones han generando el $60,5 \%$ de las estancias, correspondiendo a las mujeres el $39,5 \%$ restante.

Los ingresos, cuya duración fue de una semana o menos, generaron el $19,5 \%$ del total de las estancias y el $80,5 \%$ restante fue originado por los ingresos cuya duración superó los siete días.

El 65,3\% de las estancias correspondieron a ingresos urgentes frente al 36,5\% de los programados. El 68,9\% de los ingresos con menos de una semana de estancia son generados por ingresos programados, mientras el $61,1 \%$ de los ingresos que duran más de una semana son generados por ingresos urgentes, siendo estas diferencias estadísticamente significativas. La media de días de estancia para ingresos programados fue de 7,3 días mientras para los urgentes se sitúa en 12,5.

\section{Análisis de la inadecuación}

Se ha abordado la inadecuación desde dos puntos de vista basándonos en la bibliografía publicada ${ }^{13,14}$; según el ingreso, catalogándolo como adecuado si todos los días del mismo son adecuados; y en función de la estancia, evaluando la adecuación de cada uno de los días del ingreso por separado.

Así, se ha observado que el $54,0 \%$ de los ingresos presentaron al menos un día de estancia inadecuada. La media de días de inadecuación por ingreso se sitúa en 3,4 $(\mathrm{DE}=5,58)$, y la mediana en un día de inadecuación por ingreso.

La tasa de inadecuación global es del $34,17 \%$ (IC 95\%; 33,28-35,08).

Las mujeres presentaron un $8 \%$ más de riesgo de presentar una inadecuación en su estancia que los hombres, RR (riesgo relativo) 1,08 (IC 95\% 1,03-1,14).

La distribución por rangos de edad de los ingresos con más de un día de estancia inadecuada y la tasa de inadecuación presentan una tendencia ascendente a medida que aumenta la edad de los pacientes. Los mayores de 65 años presentan un $20 \%$ más de riesgo de sufrir una estancia inadecuada con respecto a los menores de 45 años, RR 1,20 (IC 95\% 1,10-1,32).

Se observaron diferencias estadísticamente significativas respecto a la distribución de los ingresos con más de un día de estancia inadecuada, atendiendo al tipo de ingreso (urgente o programado), siendo más frecuente en los ingresos programados. 
La tasa de inadecuación por tipo de servicio es del $38,47 \%$ para los médicos y del $30,95 \%$ para los quirúrgicos, lo cual supone una diferencia estadísticamente significativa $(p<0,0001)$. El ingreso en un servicio médico presenta un $24 \%$ más de riesgo de presentar una estancia inadecuada $[\mathrm{RR}=1,24$ (IC 95\% 1,18-1,31)].
Es importante contrastar el porcentaje de estancias generadas por cada servicio, con el porcentaje de ingresos que ha generado cada uno. Esto está reflejado en las figuras 1 y 2 , en los que también se incluye el porcentaje de días inadecuados generados por cada servicio. Para aquellos servicios en los que el porcentaje de estancias

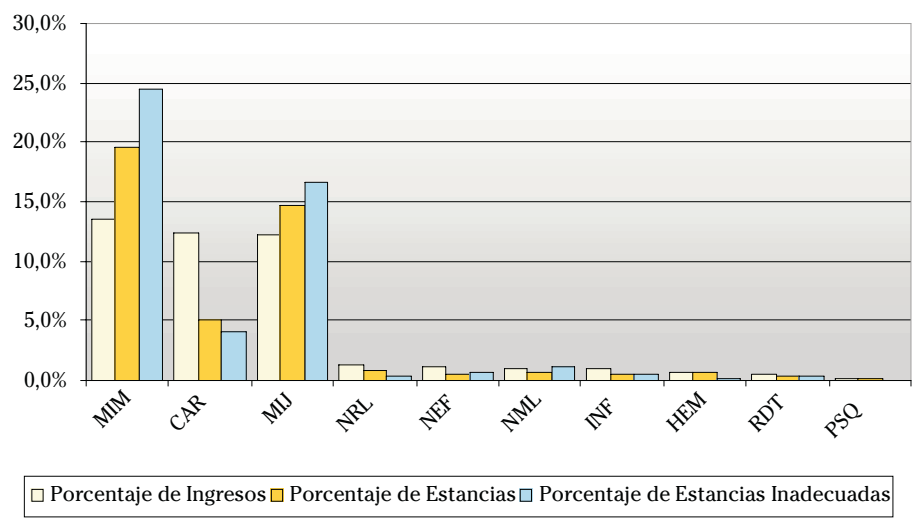

MIM: Medicina Interna I; CAR: Cardiología; MIJ: Medicina Interna II; NRL: Neurología; NEF: Nefrología; INF: Infecciosas; HEM: Hematología; RDT: Radiología; PSQ: Psiquiatría

Figura 1. Distribución de ingresos y días de estancia, global e inadecuada, por servicios médicos.

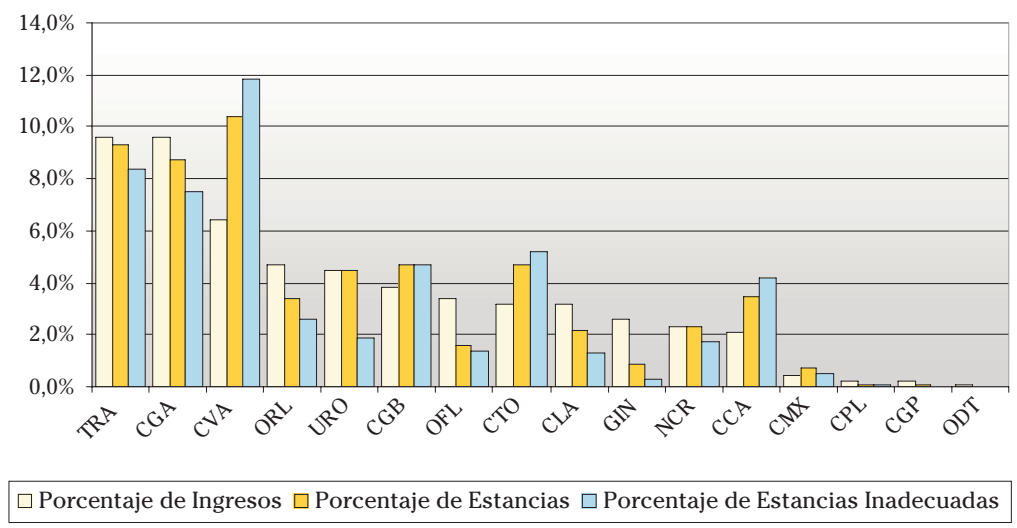

TRA: Traumatología; CGA: Cirugía General A; CVA: Cirugía Vascular; ORL: Otorrinolaringología; URO: Urología; CGB: Cirugía General B; OFL: Oftalmología; CTO: Cirugía Torácica; CLA: Cirugía Laparoscópica; GIN: Ginecología y Obstetricia; NCR: Neurocirugía; CCA: Cirugía Cardiaca; CMX: Cirugía Maxilofacial; CPL: Cirugía Plástica; CGP: Cirugía General P; ODT: Odontología

Figura 2. Distribución de ingresos y días de estancia, global e inadecuada, por Servicios quirúrgicos. 
que originan se sitúa por encima del peso que suponen del porcentaje de ingresos totales del hospital, la proporción de estancias inadecuadas es mayor De forma análoga, para aquellos servicios en los que el porcentaje de estancias es menor que el porcentaje de ingresos, la proporción de estancias inadecuadas es menor. Esta tendencia indica que a medida que la duración de la estancia es mayor aumenta la proporción de días de estancia inadecuada por encima de lo que cabría esperar, y viceversa.

La tasa de inadecuación para los diferentes servicios médicos va desde un $11,01 \%$ a un $54,95 \%$ (Tabla 1 ), y para los quirúrgicos va desde un 12,16 a un $41,96 \%$ (Tabla 2).
En los ingresos con una duración menor a una semana el 33,5\% presentaron algún día de estancia inadecuada, en contraste a los ingresos con una duración mayor de 7 días, en los que se ha observado que el $78,1 \%$ presentaron algún día de estancia inadecuada. Así, los pacientes que presentan un ingreso con estancia mayor de 7 días tienen un $86 \%$ más de riesgo de sufrir una estancia inadecuada [RR= $1,86$ IC $95 \%(1,72$ y 2,03$)]$.

Un hecho de interés al estudiar la distribución de la inadecuación de las estancias se sitúa en la descripción de la inadecuación por percentiles, ya que cuantifica la magnitud de la estancia inadecuada. El percentil $50\left(\mathrm{P}_{50}\right)$ se sitúa en un día de inadecuación por ingreso. Esto quiere decir

Tabla 1. Tasas de inadecuación por servicios. Servicios médicos.

\begin{tabular}{ccccc}
\hline Tabla 1. & Tasa de Incidencia & RR & IC (lím. inf.) & IC (lím. sup.) \\
\hline Tasa Global & 0,3417 & - & - & - \\
HEM & 0,1101 & 1,0000 & - & - \\
NRL & 0,1707 & 1,5508 & 0,7287 & 3,4578 \\
RDT & 0,2586 & 2,3491 & 1,0265 & 5,4964 \\
CAR & 0,2702 & 2,4541 & 1,3769 & 4,8219 \\
INF & 0,3867 & 3,5122 & 1,7382 & 7,5569 \\
MIJ & 0,3916 & 3,5572 & 2,0266 & 6,9095 \\
NEF & 0,3976 & 3,6114 & 1,8177 & 7,6791 \\
MIM & 0,4250 & 3,8606 & 2,2028 & 7,4902 \\
NML & 0,5495 & 4,9917 & 2,6611 & 10,1858 \\
\hline
\end{tabular}

HEM: hematología; NRL: neurología; RDT: radioterapia; CAR: cardiología; INF: infecciosas; MIJ:medicina interna II; NEF: nefrología; MIM: medicina interna I; NML: neumología.

Tabla 2. Tasas de inadecuación por servicios. Servicios quirúrgicos.

\begin{tabular}{ccccc}
\hline Tabla 2 & Tasa de Incidencia & RR & IC (lím. inf.) & IC (lím. sup.) \\
\hline Tasa Global & 0,3417 & - & - & - \\
GIN & 0,1216 & 1,0000 & - & - \\
URO & 0,1466 & 1,2055 & 0,7271 & 2,112 \\
CLA & 0,2062 & 1,6955 & 1,0017 & 3,0187 \\
NCR & 0,2595 & 2,1333 & 1,2806 & 3,7517 \\
ORL & 0,2640 & 2,1708 & 1,3263 & 3,7654 \\
CMX & 0,2778 & 2,2840 & 1,2325 & 4,3509 \\
CGA & 0,2931 & 2,4098 & 1,5058 & 4,1065 \\
OFL & 0,2943 & 2,4201 & 1,4358 & 4,2947 \\
TRA & 0,3068 & 2,5228 & 1,5785 & 4,2948 \\
CGB & 0,3385 & 2,7836 & 1,7267 & 4,7712 \\
CTO & 0,3768 & 3,0982 & 1,9253 & 5,3031 \\
CVA & 0,3887 & 3,1959 & 2,0061 & 5,4267 \\
CCA & 0,4196 & 3,4504 & 2,1359 & 5,9239 \\
\hline
\end{tabular}

GIN: ginecología y obstetricia; URO: urología; CLA: cirugía laparoscópica; NCR: neurocirugía; ORL: otorrinolaringología; CMX: cirugía maxilofacial; CGA: cirugía general A; OFL: oftalmología; TRA: traumatología; CGB: cirugía general B; CTO: cirugía torácica; CVA: cirugía vascular; CCA: cirugía cardiaca. 
que el $50 \%$ de los ingresos han presentado más de un día de inadecuación. Asimismo, el 20\% de los ingresos evaluados presentan más de 6 días de estancia inadecuada $\left(\mathrm{P}_{80}=6\right)$.

Al desagregar el ingreso por tercios, la media de días de estancia inadecuada en cada tercio presenta un incremento paulatino a medida que discurre el ingreso. Igualmente, se observa un aumento de la tasa de inadecuación a medida que avanza la estancia. En el tercer tercio la tasa de inadecuación es del 46,33\% (IC 95\% 44,44$48,29)$, lo que supone un exceso de riesgo de inadecuación del $36 \%$ respecto a la tasa de inadecuación global (primer tercio $27,61 \%$ y segundo tercio $29,77 \%)$.

\section{Análisis de las causas de inadecuación}

El análisis de las causas que generan la inadecuación se ha abordado desde dos perspectivas, por causas específicas y por bloques causales. De los 1.630 ingresos evaluados 881 presentaron algún criterio de inadecuación. De éstos, el 68,9\% presentaron al menos un criterio de inadecuación por responsabilidad del médico o del hospital; el 51,3\% presentaron al menos un criterio de inadecuación por retraso en el desarrollo del estudio o tratamiento para el que el paciente está hospitalizado; y el $3,3 \%$ presentaron al menos un criterio de inadecuación por responsabilidad social o del medio.

Como causas específicas de inadecuación las más frecuentes entre las estancias evaluadas fueron (Fig. 3):

- La realización de cualquier procedimiento diagnóstico o tratamiento necesario en el hospital pudiendo realizarse como paciente externo, que se presentó en el $31,2 \%$.

- La espera de resultados de pruebas diagnósticas o interconsultas necesarias para la toma de decisiones terapéuticas o diagnósticas, que apareció en el $28,1 \%$.

- La no existencia de un plan diagnóstico y/o tratamiento en la historia clínica, que supuso el $23,2 \%$.

- El ingreso prematuro, como criterio de inadecuación que se presentó en el $22,8 \%$.

- La permanencia del paciente en el centro cuando ya no recibe o requiere los servicios de una centro de agudos que aparece en el $21,5 \%$.

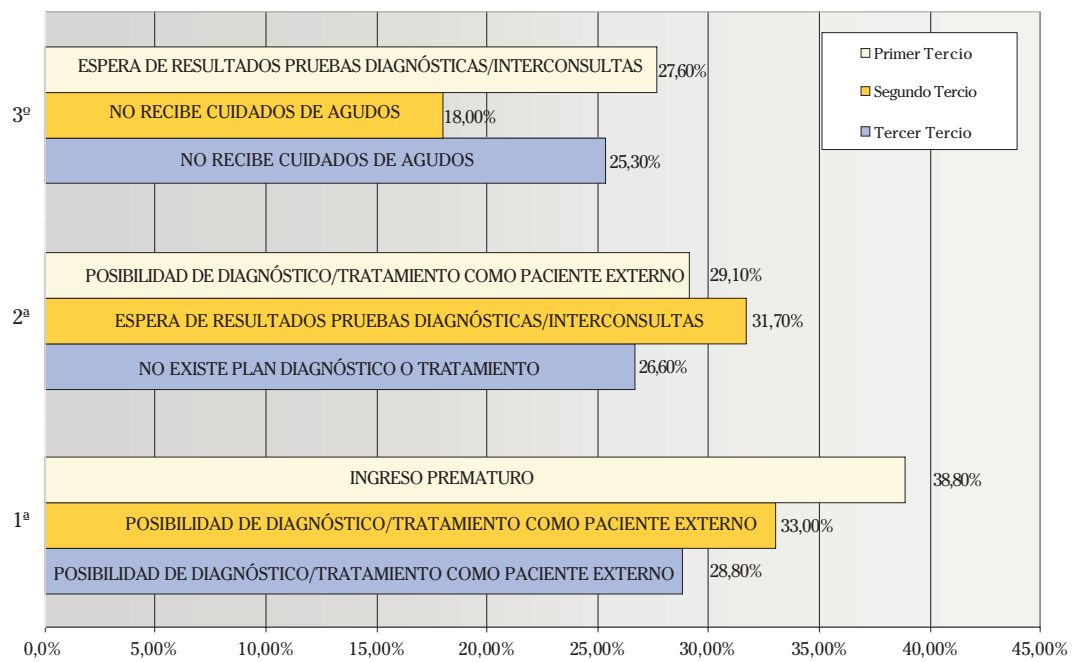

Figura 3. Distribución porcentual de las causas específicas de inadecuación por tercios de estancia. 
La distribución por tercios de ingreso de los bloques causales de inadecuación presenta semejanzas entre el segundo y el tercer tercio, siendo la estructura diferente en el primero. Esto se ve reflejado en la tabla 3 .

\section{DISCUSIÓN}

En primer lugar, hay que señalar las limitaciones inherentes al propio diseño. Puede presentarse un sesgo de información derivado de una incorrecta medida de los eventos y variables de interés. Es probable que nuestro instrumento de medida (AEP), presente una tendencia a sobreestimar la inadecuación de la estancia respecto al criterio clínico, que tiene en cuenta la visión subjetiva del profesional sanitario.

Otro aspecto a tener en cuenta, es que la catalogación de una estancia como inadecuada se realiza a través de las anotaciones recogidas en la historia clínica, esto supone, que si el facultativo no deja constancia de los parámetros que podrían justificar el ingreso del paciente, éstos no pueden ser evaluados, lo que podría sobreestimar la inadecuación. Sin embargo, un hecho que va en la dirección contraría es que no se valora la indicación de dichos criterios, es decir, se acepta que todas las decisiones diagnósticas y terapéuticas son correctas, con independencia del nivel donde se prestan y el momento o tiempo de su presentación.

Puede haber otros factores que influyen en la inadecuación de la estancia, como la presión de la familia, la percepción personal del estado de salud, las con- diciones sociales, etc., que no están recogidos en la historia clínica y que pueden estar actuando como factores de confusión. En este sentido, un aspecto destacable es que las condiciones sociales del paciente no están reflejadas en la historia clínica. Como resultado, es probable que estén infraestimadas como causantes de inadecuación. También hay que precisar que los riesgos de inadecuación calculados son crudos, y que sería preciso realizar un análisis estratificado o multivariante para valorar la confusión o la posible interacción.

Una recogida activa de la información y una correcta codificación del diagnóstico y de los parámetros que determinan la complejidad de los pacientes, podría facilitar el ajuste por estas variables y dar como resultado unas estimaciones más precisas.

A la luz del los resultados del estudio se puede concluir que la tasa de inadecuación está relacionada con la duración de la estancia, es decir, la relación entre la inadecuación y la estancia no es lineal, sino que aumenta mucho al incrementarse los días de estancia. Además, tanto el sexo como la edad son factores que influyen en la aparición de inadecuación. Por otra parte, es más frecuente que aparezca inadecuación en los ingresos con una duración mayor de 7 días de estancia y en los ingresos programados, apareciendo con mayor frecuencia la inadecuación en los servicios médicos.

La instauración de la informatización de la historia clínica única se hace necesaria como herramienta para la mejora de la

Tabla 3. Distribución porcentual de las causas de inadecuación por bloques causales.

\begin{tabular}{clr}
\hline Tercios & \multicolumn{1}{c}{ Bloques causales } & Porcentaje \\
\hline \multirow{2}{*}{ PRIMERO } & Retraso en el desarrollo del estudio o tratamiento & $66,8 \%$ \\
& Responsabilidad del médico o del hospital & $41,9 \%$ \\
& Responsabilidad social o del medio & $1,6 \%$ \\
\cline { 2 - 3 } & Retraso en el desarrollo del estudio o tratamiento & $64,1 \%$ \\
SEGUNDO & Responsabilidad del médico o del hospital & $44,9 \%$ \\
& Responsabilidad social o del medio & $3,8 \%$ \\
\cline { 2 - 3 } & Retraso en el desarrollo del estudio o tratamiento & $79,5 \%$ \\
TERCERO & Responsabilidad del médico o del hospital & $22,9 \%$ \\
& Responsabilidad social o del medio & $3,4 \%$ \\
\hline
\end{tabular}


gestión clínica y es necesario hacer hincapié en la recogida de los factores sociales asociados a la situación del paciente en la historia clínica.

La utilización de métodos de identificación del uso inapropiado como el AEP presenta aplicaciones tanto en planificación como en gestión hospitalaria, al permitir identificar problemas hospitalarios causantes de demoras, principalmente problemas de tipo organizativo, permitiendo el desarrollo de intervenciones encaminadas a la reducción del uso inapropiado.

La mejora de la calidad asistencial como consecuencia no sólo de una menor probabilidad de infección nosocomial, al reducir el número de estancias innecesarias, si no también de una reducción en las listas de espera pueden ser otras de las consecuencias derivadas de la utilización del AEP.

\section{BILBIOGRAFÍA}

1. Peiró S, Portella E. Identificación del uso inapropiado de la hospitalización: la búsqueda de la eficiencia. Med Clin (Barc) 1994; 103: 65-71.

2. Fetter RB, Shin Y, Freeman JL, Averil RF, Thompson JD. Case-Mix. Definition by diagnosis related groups. Med Care 1980; 18: 1-53.

3. Peiró S, Meneu R, Roselló ML, Martínez E, Portella E. ¿Qué mide la estancia media de los grupos relacionados con el diagnóstico? Med Clin (Barc) 1994; 103: 413-417.

4. Gertman PM, RESTUCCIA JD. The appropriateness evaluation protocol: a technique for assessing unnecessary days of hospital care. Med Care 1981; 19: 855-871.

5. REstuccia JD. Appropriateness Evaluation Protocol. Manual para la revisión. Barcelona: Fundación Avedis Donabedian, 1984.
6. Peiró S, Meneu R, Roselló Ml, Pontella E, CARbonell R, Fernández $\mathrm{C}$ et al. Validez del protocolo de evaluación de uso inapropiado de la hospitalización. Med Clin (Barc) 1996; 107: 124-129.

7. Antón P, Peiro S, Aranaz JM, Calpena R, ComPAN A, LEUTSCHER E. Uso inapropiado de la hospitalización en cirugía general. Magnitud, factores asociados y causas. Cir Esp 2005; 78: 183-191.

8. Callejas Jl, Fernández A, Palmero C, Navarro MD. ¿Son realmente objetivos los criterios de adecuación hospitalario del Appropriateness Evaluation Protocol (AEP). Med Clin (Barc) 2002; 118: 157-158.

9. Strumwasser I, Paranjpe NV, Ronis DL, Share D, SELL LJ. Reliability and validity of utilization review criteria. Appropriateness Evaluation Protocol, Standardized Medreview Instrument, and Intensity-Severity-Discharge criteria. Med Care 1990; 28: 95-111.

10. Rishron S, Rishron S, Lubacsh S, Epstein LM. Reliability of a method of determining the necessity for hospitalization days in Israel. Med Care 1986; 24: 279-282.

11. Fuente D, Peiró S, Portella E, Marchan C, AYMERICH S. Utilización innecesaria de la hospitalización: Importancia de la gestión a nivel de Serv Rev Cal Asist 1994; 1: 8-16.

12. Ochoa J, Villar A, Ramalle E, Carpintero JM, Bragado L, RUIZ JI. Adecuación de los ingresos hospitalarios urgentes. Ann Med Interna 2002; 19: 446-448.

13. Negro JM, Guerrero M, Ferrándiz R. El Protocolo de Evaluación del Uso Inapropiado de la Hospitalización (The Appropriateness Evaluation Protocol) en Alergología. Rev Esp Alergol Inmunol Clin 1998; 13: 171-180.

14. Mirón JA, Orosco JA, Alonso M, SÁEnz MC. Estimación de ingresos y estancias inadecuadas en un hospital comarcal. Rev Clin Esp 2000; 12: 654-658. 